\title{
Evaluation of Llaima volcano activities for localization and classification of LP, VT and TR events
}

\author{
Ali Dehghan Firoozabadi ${ }^{*}$, Fabian Seguel ${ }^{* *}$, Ismael Soto ${ }^{* *}$, David \\ Guevara $^{* *}$, Fernando Huenupan ${ }^{* * *}$, Millaray Curilem ${ }^{* * *}$, Luis Franco ${ }^{* * * *}$
}

\begin{abstract}
Evaluation of seismic signals is one of the most important research topics on Volcanology. Volcanoes have daily activity; therefore, high speed evaluation of recorded signals is a challenge for improving the study of the natural phenomena occurring inside these natural formations. The aim of this paper is the evaluation (denoising, localization and classification) and analysis of Llaima volcano activities, one of the most actives volcanoes in South America. Different already proposed methods, such as, Butterworth, Spectral Subtraction (SS) and Wiener Filter (WF) are compared to the proposed Modified Spectral Subtraction (MSS) and Modified Wiener Filter (MWF) to find the best method for denoising the volcano signals. Then, event localization based on received signals of volcano is performed. In this step, Time Delay Estimation (TDE)-based method is used on data acquired from 3 mechanical sensors located in the volcano area. The proposed method is used to estimate the area for event location. The proposed denoising methods make the starting point for the event more evident to increase the localization accuracy for events where the starting point is difficult to find. In the last step, a method based on the novel DNN technique is proposed to classify the three main events occurring in the Llaima volcano (TR (Tremor), LP (Long Period) and VT (Volcano Tectonic)).
\end{abstract}

K e y w o r d s: seismic signals, Llaima volcano, spectral subtraction, wiener filter, deep neural network, source localization

\section{Introduction}

Chile is considered as a natural laboratory, where huge earthquakes and volcanic eruptions occurs frequently. This is because the subduction between the Nazca and South American plates $[1,2]$. This subduction of the oceanic cortex below the continental margin has allowed the development of the major orogenic system called "Los Andes" [3]. Chile has a sizeable portion of this surface compared with other countries. Earthquakes and volcanic eruptions affect the inhabitant's safety and the infrastructure due to the proximity of the cities to the threatening sites.

Chile has around 100 actives volcanoes including individual stratovolcanoes and groups of monogenetic volcanoes [4]. The main active volcanoes are located in the continent, forming the Andean volcanic belt. Based on the historic record, 61 of those volcanoes have had more than 400 eruptions in the last 500 years. Natural disasters, particularly the disasters caused by volcanic eruptions have taken the attention of the authorities and the scientific community. Improving the emergency response and predict the volcano eruption in previous stages has become a matter of major concern.

Nowadays the Servicio Nacional de Geologia (Sernageomin) by means of the Observatorio Vulcanológico de los Andes del sur [5] is monitoring 43 actives volcanoes which are considered as the most actives and dangerous volcanoes in the country. About the $16 \%$ Llaima volcano belong to the Andean volcanic belt and it is one of the most active and massive volcanoes in South America [6]. It is located in the Región de La Araucanía at coordinates $38.698^{\circ} \mathrm{S}$ and $71.730^{\circ} \mathrm{W}$ and it is classified as a stratovolcano [7]. Seismic sensors placed nearby the volcano are used to record signals, these are MOT $\left(38.675^{\circ} \mathrm{S}\right.$ and $\left.71.784^{\circ} \mathrm{W}\right)$, LAV $\left(38.705^{\circ} \mathrm{S}\right.$ and $\left.71.649^{\circ} \mathrm{W}\right)$ and LLA $\left(38.774^{\circ} \mathrm{S}\right.$ and $\left.71.696^{\circ} \mathrm{W}\right)$. These seismic sensors are equipped with seismometers with a bandwidth of $50 \mathrm{~Hz}$ in $\mathrm{X}, \mathrm{Y}$ and $\mathrm{Z}$ directions. The data recorded about the seismic events is sent in real time to the OVDAS [8].

There are many possible reasons that gives origin to a seismic event. Fault fractures and eruptive activity generate different seismic events. According to their origin, seismic events can be classified as Body Waves in which Primary wave (P) and Secondary waves (S) can be found and Surface Waves where Rayleigh wave (R) and Love Wave (L) are grouped.

Volcanoes produce a wide variety of signals produced by the magma flowing and hydrothermal fluids interacting with solid rocks [9]. Events caused by the fracture of brittle material due to changes produced by the flow of magma or fluids are called Volcano-Tectonic (VT). Oscil-

* Department of Electricity, Universidad Tecnologica Metropolitana, Av. Jose Pedro Alessandri 1242, Santiago, Chile, adehghanfirouzabadi@utem.cl, ** Electrical Engineering Department, Universidad of Santiago of Chile, Santiago, 9170124, Chile, fabian.seguelg@usach.cl, ismael.soto@usach.cl, david.guevara@usach.cl, *** Electrical Engineering Department, University of Frontera, Temuco, Chile, fernando.huenupan@ufrontera.cl, gloria.curilem@ufrontera.cl, **** Observatorio Vulcanolgico de Los Andes del Sur (OVDAS); Santa Maria 0104, Providencia, Chile, luis.franco@sernageomin.cl 


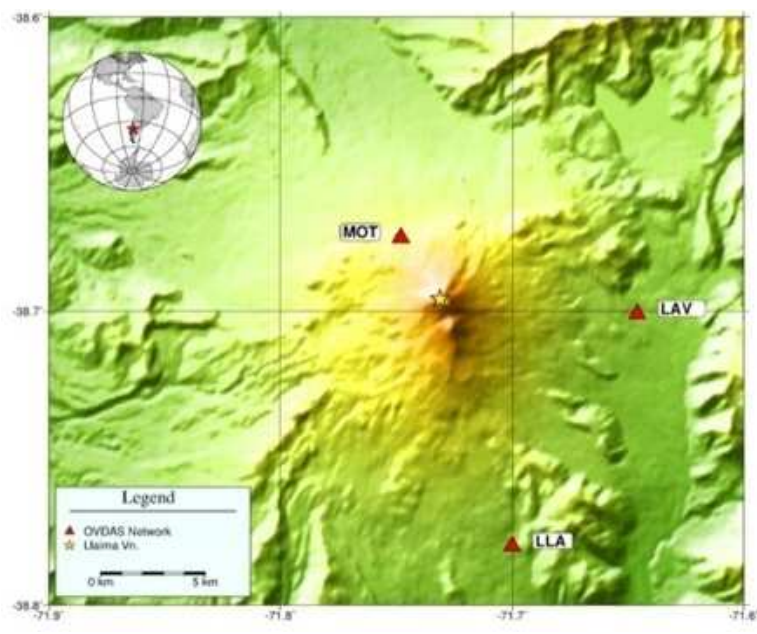

Fig. 1. Top view of Llaima volcano with MOT, LAV and LLA sensors $[8]$

lating changes in the pressure produced by the dynamics of the fluids inside the ducts or small fissures are denominated Long Period (LP) and TRemor (TR) events. Long-Period signals (LP) are usually low frequency events whose duration is bounded by a range from seconds to minutes. The bandwidth of this event is $4 \mathrm{~Hz}$ located in the range of $1 \mathrm{~Hz}$ to $5 \mathrm{~Hz}[10]$. However, they are often preceded by small energy movements of frequencies greater than $5 \mathrm{~Hz}$. Another classification group of the seismic events is Volcano Tectonic (VT). These waves may last from seconds to minutes [11] and are characterized for having $\mathrm{P}$ waves at the beginning of the signal followed by $\mathrm{S}$ waves. Both are clearly defined in the signal graph. The bandwidth of this kind of events is variable and the frequency is mainly focused between $2 \mathrm{~Hz}$ and $15 \mathrm{~Hz}$. Finally, Tremor events (TR) are constant vibrations with a frequency range from $1 \mathrm{~Hz}$ to $5 \mathrm{~Hz}$ and its duration can vary from minutes, days and even months [12]. Figure 1 shows the location of the Llaima volcano and the distribution of the sensors around the active crater. Also, Tab. 1 shows position for MOT, LAV and LLA sensors.

Table 1. The position for MOT, LAV and LLA sensors

\begin{tabular}{lccc}
\hline & Code & Lat (0) S & Long (0) S \\
\hline Motion & MOT & 38.675 & 71.784 \\
Laguna Verde & LAV & 38.705 & 71.649 \\
Llaima & LLA & 38.774 & 71.696 \\
\hline
\end{tabular}

In this paper the aim is localization and classification of TR, VT and LP events in Llaima volcano. For this purpose, one of the most important steps is preprocessing the obtained signal. Received signals from MOT, LAV and LLA have a high level of noise. Noise is divided to two main groups: 1) White noise with uniform frequency spectrum, and 2) Colorful noise with low frequency spectrum. For filtering the signal, a comparison between some denoising methods applied on volcano signals is performed. From the denoising process high quality signals will be obtained: Denoised signals will be used to perform the localization and classification of the events. Therefore, the proposed block diagram in this paper can be shown as Fig. 2.

The signal enhancement tries to improve the quality of signal to increase the performance of localization and classification. Several methods have been proposed for signal enhancement such as: Butterworth filter [13], Spectral Subtraction [14], Statistical model based methods (Hidden Markov Model) [15], sub-space based methods [16], etc. These methods have a high performance when dealing with uniform spectrum and additive noise. In this paper, two of the most effective filters, Spectral Subtraction (SS) and Wiener Filter (WF), will be applied on the recorded signals. Also, Modified Spectral Subtraction (MSS) and Modified Wiener Filter (MWF) will be implemented to improve the performance of denoising stage. SS and WF have an appropriate performance when dealing with stationary uniform spectrum noise. However, recorded signals of volcano events have colorful noise with low frequency components. Butterworth filter (BW) could remove the noise in the event's frequency range. Because of this, Butterworth filter with cut-off frequency $30 \mathrm{~Hz}$ is used in a preprocessing step before using SS, WF, MSS and MWF.

Once the signal has been enhanced, a low complexity localization method with high accuracy to detect the event's location is implemented. Events happen in the depth of the earth. Mechanical waves of the events arrive to the surface after crossing different earth layers where can be recorded by the sensors. Useful information about the volcano can be obtained by means of event's localization. The most common method used in OVDAS and in some other volcano logical observatories is the modified HYPO71. This technique determines earthquake locations and magnitudes from seismic network data like first-arrival $\mathrm{P}$ and $\mathrm{S}$ arrival times, amplitudes and coda

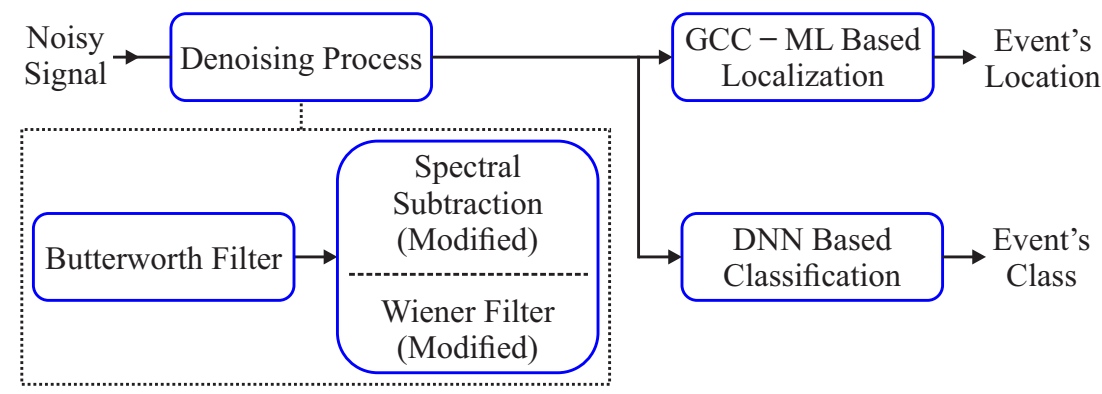

Fig. 2. Block diagram of proposed Llaima Volcano signal processing 
durations [17]. Also, There are some improvements to HYPO71, they can be found in $[18,19]$.

Source localization algorithms are divided to one-stage and two-stages methods [20]. Two-stages methods estimate the time difference of arrival (TDOA) between each pair of sensors. Then the location of events is estimated based on TDOA values and sensor's location. On the other hand, in one-stage methods a cost function is calculated for candidate points. Then, the more likely point is selected as source location. Two-steps methods (based on TDOA) are faster than one-step methods (based on energy). Time delay of the signal is estimated using low complexity equations in two-stages methods. On the other hand, in one-stage methods its computational complexity is directly depends on the number of candidate points. One of the main factors that causes detrimental on the localization estimation in TDE-based methods is the reverberation of the signal. Nevertheless, the physical medium in which the event's mechanical waves are propagated can be assumed as free space, ie there is no reverberation. Because of this, a high performance on the event localization can be achieved by using crosscorrelation based methods.

Localization methods are divided into parametric and non-parametric groups. Parametric methods [21] usually are beam forming or Maximum Likelihood (ML) methods which define a spatial maximum likelihood function for event's positions. Each function can have multiple maximum in the searching space, making the selection of the maximum a high complexity process. Non-parametric methods [22] are based on signal sub-space or Eigen value analysis. For example, MUSIC [23] and ESPRIT [24] are two important methods in non-parametric group that have higher resolution than parametric methods. These methods are designed for using multiple sensors and narrow band signals. In addition to this, some research work have been done to extend these methods for wide band signals [25].

In this paper, GCC method is used for localization of different kind of events [26]. This method has appropriate accuracy and it can be used for real-time application. Two weighted functions are usually used in combination with GCC, these are: 1) PHAT (PHAse Transform) and, 2) ML weighted function. PHAT and ML weighted functions are suitable for signals affected by reverberation and noise respectively. Since the major adverse factor in this application is noise, GCC is used in combination with ML weighted function.

The feature extraction is one of the most important aspects in pattern recognition and classification problems since the selection of the features can improve or deteriorate the performance of the system. Most of the approaches for classification in volcano signal processing has been made by selecting the spectral based features in a hand-crafted method which is subjective to perception of the designers and the optimal set of features is not always reached. Once the selection of the features is made, there are several techniques for the classification, in particular, techniques from the field of artificial intelligence (such as Support Vector Machines (SVM) and Artificial Neural Networks (ANN)) has been widely applied for many classification applications such as classification of cancel cells [27] real time electro cardiogram classification [28], sound event classification [29], electrical disturbances [30], internet traffic [31]. Support Vector Machines or Kernel machines and neural networks with one hidden layer have a shallow architecture, namely, two levels of data-dependent computational elements [32]. Recently, a major advance has been the ability to train deep architectures of neural networks, that is, networks with many weights to adapt. The main issue with "deep" architectures is the ability of the training algorithm to propagate the error and find the best set of weights that minimize the error. Deep Learning tries to overcome this problem by first pre-training the network layer by layer by means of an unsupervised learning algorithm. This pre-training tries to build an internal representation of the data reducing the dimensionality [33]. Once the weights are initialized a fine-tuning stage is performed by means of a supervised learning algorithm using labeled data. Deep Neural Networks has achieved promising result when used in for several applications such as acoustic emotion recognition [34], speech recognition [35], oceanographic object classification [36] and so on. In this paper we propose the classification of volcano signals by means of a DNN based classifier.

After introducing SS and WF, we propose MSS and MWF to improve the methods. The details of GCC and ML function is introduced, and explained is the way of using GCC method for this sensor structure. We examine denoising methods based on 3 sensors to find the best method for recorded signals enhancement and use enhanced signals for localization. The results are shown for GCC method in combination with ML weighted function. Finally, DNN-based classification method is also shown.

\section{Methodology}

The methods used for signal enhancement, event localization and classification will be introduced here. First, several common denoising methods will be reviewed to find the best method for denoising the signals obtained by the mechanical sensors placed on volcano surroundings. Then Time Delay Estimation (TDE)-based method for localization of event will be introduced. Finally, a method based on the novel DNN technique is proposed to classify the three main events occurring in the Llaima volcano.

\subsection{Signal Enhancement}

As it was mentioned in previous section, the aim of this paper is localization and classification of recorded events from Llaima volcano. A preprocessing stage in which the noise is removed from the signal must be done because of the high level of noise. In this section, first SS and WF methods are introduced. Then, MSS and MWF will be proposed to improve traditional methods. 


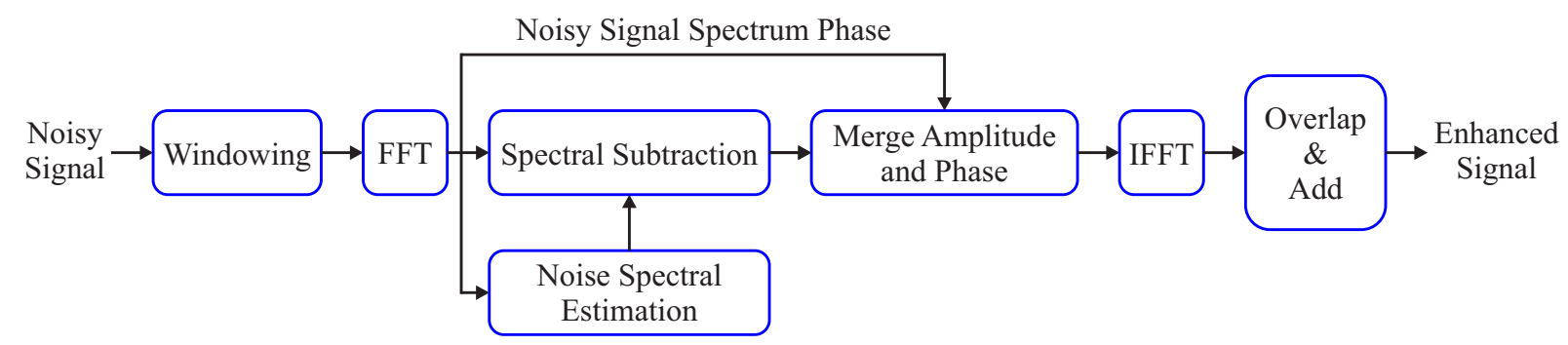

Fig. 3. The block diagram for Spectral Subtraction method

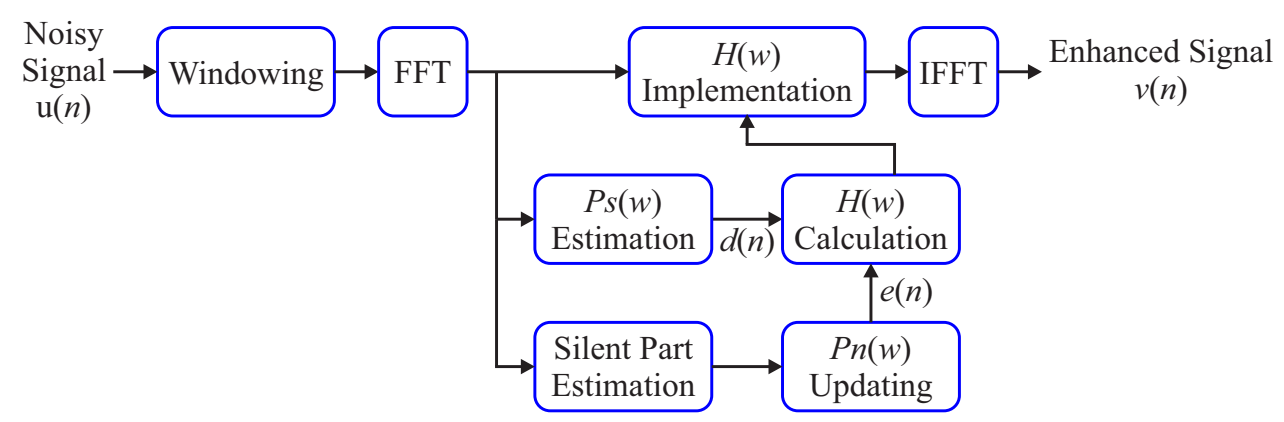

Fig. 4. The block diagram for Wiener filter implementation

\subsubsection{Spectral subtraction (S S) method}

Spectral Subtraction is a method for power spectral retrieval of signal that has been affected by additive noise. The idea in SS method is based on estimation the amplitude for frequency spectrum. This estimation can be achieved by subtraction between the amplitude of noise spectrum and noisy signal spectrum. The information related to noise spectrum is estimated by using the silent part of noisy signal. SS block diagram has been shown in Fig. 3.

Firstly, recorded signals by sensors are windowed with $50 \%$ overlapping. Then, the Fourier transform is implemented on each frame of signal. Noise spectral estimation is an important part in this method. These noise estimations are achievable of free-event part of signals. Then recorded signals are divided to equal part with length $L$ by windowing and are transferred to frequency domain with Fast Fourier Transform (FFT) function. Windowed signal can be expressed in frequency domain as

$$
Y_{w}(\omega)=W(\omega) * Y(\omega)=S_{w}(\omega)+N_{w}(\omega)
$$

where $Y_{w}(\omega), S_{w}(\omega)$ and $N_{w}(\omega)$ are Fourier transform for windowed noisy signal, windowed clean signal (event), and windowed noise respectively and $*$ is convolution operator. Also $Y(\omega)$ and $W(\omega)$ are Fourier transform for noisy signal and window. Finally, Spectral Subtraction can be expressed as

$$
\left|\hat{S}_{w}(\omega)\right|^{2}=\left|Y_{w}(\omega)\right|^{2}-E\left\{\left|N_{w}(\omega)\right|^{2}\right\} .
$$

In $(2),\left|\hat{S}_{w}(\omega)\right|^{2}$ is the spectral subtraction for a clean signal and $E\left\{\left|N_{w}(\omega)\right|^{2}\right\}$ is the expected value of the spectral noise on the silent frames.
Finally, enhanced signal can be expressed as the amplitude of estimated spectral and noisy signal phase as

$$
\hat{s}_{w}[n]=F^{-1}\left\{\left|\hat{S}_{w}(\omega)\right| \exp (j \arg (Y(\omega)))\right\}
$$

where $F^{-1}$ is the inverse Fourier transform and $\hat{s}_{w}[n]$ is enhanced signal for a frame (window).

\subsubsection{Wiener Filter (W F) for signal d e no is ing}

$\mathrm{WF}$ is a common method for noise reduction. This method decreases the noise of noisy signal by statisubtical estimation of the noise in time domain. In WF, a filter is designed based on signal and noise parameters. Noisy signal is crossed with the filter in order to decrease the noise level. Then, a new filter is designed based on parameter of enhanced signal in the previous step. This process repeated until the enhanced signal is obtained [37].

Preprocessing is needed to prepare noisy signal for WF algorithm. Figure 4 shows block diagram for WF implementation. Wiener Filter transfer function can be written as

$$
H(\omega)=\frac{P_{s}(\omega)}{P_{s}}(\omega)+P_{n}(\omega)
$$

where $H(\omega), P_{s}(\omega)$ and $P_{n}(\omega)$ are impulse response of WF, power spectral density for clean signal and noise respectively. Noise power spectral can be estimated from silent part of signal. Nevertheless, compute the power spectral estimation for clean signal is a difficult task.

\subsubsection{Modified Spectral Subtraction (M S S) method}

Some parameters can be modified in spectral subtraction method to obtain better performance in noise reduc- 
tion process. It means that the noise effect in subtraction operation can be changed. It should be noted that, increasing the effect of noise estimation can cause a detrimental effect on the event's information. According to this, Modified spectral subtraction can be written as

$$
\left|\hat{S}_{w}(\omega)\right|^{\alpha}=\left|Y_{w}(\omega)\right|^{\alpha}-\beta E\left\{\left|N_{w}(\omega)\right|^{\alpha}\right\}
$$

where in this equation, $\alpha$ is attenuation factor and $\beta$ is over-subtraction factor. The value for $\beta$ in this equation has to be selected bigger than 1 for over-subtraction assumption. This means upper band estimation of noise. The attenuation factor $\alpha$ has to be selected between 0 and $2(0<\alpha<2)$. A large amount of noise remains with $\alpha=2$ and with $\alpha$ selected close to 2 produce the decrement of the signal amplitude. The value of $\alpha$ and $\beta$ have to be selected in order to minimize adverse effects on the signal.

\subsubsection{Modified Wiener Filter (M W F) method}

In Wiener filter method, filters are designed based on the output signal and they are implemented on input signal repeatedly. The parameters for WF are obtained based on signal and noise power spectral estimations. The equation for modified wiener filter can be written as

$$
H_{M W F}(\omega)=\left[\frac{P_{s}(\omega)}{P_{s}(\omega)+\gamma P_{n}(\omega)}\right]^{\delta}
$$

where $\gamma$ is parameter of noise effect in filter design. The noise effect in filter design can be increased by increasing the value of this parameter. Another parameters is $\delta$ which controls the effect of clean signal spectral estimation. Is this parameter is overvalued a part of the original signal could be eliminated making the filter unsuitable. The parameters values can be considered as $\gamma>1$ and $0<\delta<2$ in order to yield a correct output.

\subsection{Generalized Cross Correlation (GCC) function for event localization}

Generalized Cross Correlation is a useful method for TDOA estimation between two sensors. The source location can be obtained by estimating the TDOAs between multiple sensor pairs. Figure 5 shows an example of delay between sensors on event location for three sensors $(m=1,2,3)$. The relation between distance and propagation delay can be written as $\tau_{m}=\frac{r_{m}^{(s)}}{c}$ [26] where $r_{m}$ is delay between event location and sensor $m, r_{m}^{(s)}$ is distance between event location and sensor $m$ and $c$ is the wave speed in stone. The variable $\tau_{l q}$ shows the TDOA between sensors $l$ and $q$ and it can be expressed as a difference between propagation delays as $\tau_{l q}=\tau_{l}-\tau_{q}$.

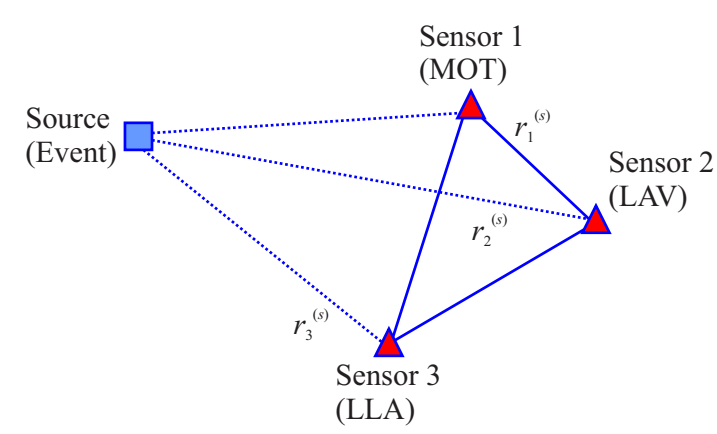

Fig. 5. Parametrization of event location by TDOA estimations

TDOA estimations can be expressed as a function of distance between the event and sensor location. This can be expressed mathematically as $\tau_{l q}=\frac{r_{l}^{(s)}-r_{q}^{(s)}}{c}$. Then, TDOAs parameterize the event location and we can localize the place of event with a number of TDOAs. Sensor signal model can be written in real condition for sensors $l$ and $q$ as $x_{l}[n]=\frac{1}{r_{l}} l s\left[n-\tau_{l}\right]+n_{l}[n]$ and $x_{q}[n]=\frac{1}{r_{q}}\left[n-\tau_{q}\right]+n_{q}[n]$ where $x[n]$ is the received signal by sensors ( $l$ or $q), n[n]$ is additive noise and $s[n]$ is transmitted signal in event location. GCC function has a peak when shifted samples of $s[n]$ are match to $\tau_{l q}$. The Fourier transform of cross correlation function can be expressed as cross spectral density between signals

$$
C_{l q}(\omega)=X_{l}(\omega) X_{q}^{\prime}(\omega)
$$

where $C_{l q}(\omega)$ is Fourier transform of cross-correlation function, $X_{l}(\omega)$ is Fourier transform of $x_{l}[n]$ and $X_{q}^{\prime}(\omega)$ is the complex conjugate of Fourier transform for $x_{q}[n]$.

Generalized Cross-Correlation function $\left(R_{l q}(\tau)\right)$ is the cross-correlation between filtered version of $x_{l}[n]$ and $x_{q}[n]$. GCC function can be expressed by Fourier transform of these filters $\left(G_{l}(\omega), G_{q}(\omega)\right)$ as

$$
R_{l q}(\tau)=\frac{1}{2 \pi} \int_{-\infty}^{+\infty}\left(G_{l}(\omega) X_{l}(\omega)\right)\left(G_{q}(\omega) X_{q}(\omega)\right)^{\prime j \omega \tau} \mathrm{d} \omega .
$$

The GCC function can be rewritten by frequency-based weighted function by using $\psi_{l q}(\omega)=G_{l}(\omega) G_{q}^{\prime}(\omega)$.

The Generalize cross-correlation function between sensors $l$ and $q$ has a peak in range of $\tau(\tau \in D)$ based on TDOA between these two sensors. In fact, TDOA is time difference that can maximize $R_{l q}(\tau)$ as $\hat{\tau}_{l q}=$ $\arg \max R_{l q}(\tau)$ where $D$ is the space for all of possible directions. Equation (8) has multiple local maximum. The amplitude and distance between these maximums are related to some factors such as the separating distance between sensors, the type of events, the noise signal and the type of weighted function $\psi_{l q}(\omega)$.

ML weighted function is an unbiased and effective estimator in non-reflection conditions and by uncorrelated noise and signals. ML weighted function can be shown as power spectral density (PSD) of event $(S(\omega))$ and PSD of noise in sensors $l\left(V_{l}(\omega)\right)$ and $q\left(V_{q}(\omega)\right)$ as in [38]

$$
\psi_{l q}(\omega)=\frac{|S(\omega)|}{\left|V_{l}(\omega)\right|\left|V_{q}(\omega)\right|}\left\{1+\frac{|S(\omega)|}{\left|V_{l}(\omega)\right|}+\frac{|S(\omega)|}{\left|V_{q}(\omega)\right|}\right\}^{-1} .
$$


It has been shown that a smooth reflection can decrease the performance of ML weighted function extremely. An approximation for this weighted function that works well on short frames of events can be rewritten as PSD of sensors signals and noises [38]. $\left|X_{l}(\omega)\right|$ and $\left|X_{q}(\omega)\right|$ are Fourier transform of sensors signals $\left|V_{l}(\omega)\right|$ and $\left|V_{q}(\omega)\right|$ are Fourier transform of additive noise that can be estimated in silent part of recorded signals. The combination between GCC and ML weighted function is called GCC-ML. The GCC-ML function has appropriate performance in noisy condition as the same condition for recorded signals in this paper.

\subsection{Deep Neural Networks (DNN) for event classifica- tion}

DNNs contain multiple hidden layers, each hidden layer is connected with the next. The DNN architecture used in this paper is shown in Fig. 6. The DNN takes the first 2000 values of the magnitude of the Fourier transform in order to obtain from these features the hidden representation of the signals.

First the DNN is trained layer by layer with an unsupervised learning algorithm (Auto encoder), then, when the unsupervised stage is accomplished the DNN is finetuned by using the back propagation algorithm.

Auto encoders is one type of unsupervised neural network. The auto encoder network firstly transforms highdimensional space data into a low-dimensional space by means of an encoder extracting some features from the input. This procedure reduces the dimensionality and, subsequently, the complexity of the network. Once the data is encoded a decoder network reconstructs the inputs from the corresponding coded data.

The input signal $x^{m} \in \mathbb{R}$ form a dataset of length $M:\left\{x^{m}\right\}_{m=1}^{M}$, which is the features input for the first layer of the network, is represented by a coded version named as $h^{m}$ where

$$
h^{m}=f_{\theta}\left(x^{m}\right) .
$$

Correspondingly the decoded version of the inputs $\hat{x}^{m}$ can be expressed as

$$
\hat{x}^{m}=g_{\theta^{\prime}}\left(h^{m}\right) .
$$

The parameters set $\left(\theta\right.$ and $\left.\theta^{\prime}\right)$ of the encoder and decoder are learned on the task of reconstructing the original input minimizing the error between the features and the reconstructed features $L(x, \hat{x})$ where $L(\cdot)$ is a loss function between $x$ and $\hat{x}$. The above explanation is mathematically proposed by

$$
\min \frac{1}{M} \sum_{m=1}^{M} L(x, \hat{x})
$$

where the loss function $L(\cdot)$ is

$$
L\left(x^{m}, \hat{x}^{m}\right)=\left\|x^{m}-\hat{x}^{m}\right\|^{2} .
$$

The reconstruction of the features $\hat{x}^{m}$ is obtained by

$$
\begin{aligned}
\hat{x}^{m} & =g_{\theta^{\prime}}\left(f_{\theta}\left(x^{m}\right)\right), \\
f_{\theta}\left(x^{m}\right) & =n_{f}\left(W x^{m}+b\right), \\
g_{\theta^{\prime}} T\left(x^{m}\right) & =n_{g}\left(W^{T} x^{m}+d\right)
\end{aligned}
$$

where $n_{f}$ and $n_{g}$ are the encoder and decoder activation functions. In this paper we use $n_{g}=n_{f}=\frac{1}{1-e^{x}}$ which is the sigmoid function. The parameters set $\theta=\{W, b\}$ and $\theta^{\prime}=\left\{W^{T}, d\right\}$ are the weight and bias matrices of the encoder and decoder respectively.

This formulation can be used to pre-train an N-layered neural network. Given an initial input signal of features data set $\left\{x^{m}\right\}_{m=1}^{M}$ in the input layer, the first encoder is trained to obtain the coded version of the initial features. Once the first layer is trained, the coded features are now used as the inputs of the next layer. The inner representation of the initial features is represented as

$$
h_{1}^{m}=f_{\theta_{1}}\left(x^{m}\right) .
$$

Since the coded features are the input data for the next layer the $N$-th encode vector of the features matrix is represented as

$$
h_{N}^{m}=f_{\theta_{N}}\left(h_{N-1}^{m}\right)
$$

where $\theta_{N}$ is the parameter set of the $N$-th auto encoder. The pre training held to the DNN to obtain a better local minimum compared to the random initialization of the weights and achieves better generalization performance in classification problems [32]. After the pre-training is performed the output targets for the classification task are used in a fine-tuning training stage. The output of the DNN for the input signal $x^{m}$ is given by

$$
y^{m}=f_{\theta_{N+1}}\left(h_{N}^{m}\right)
$$

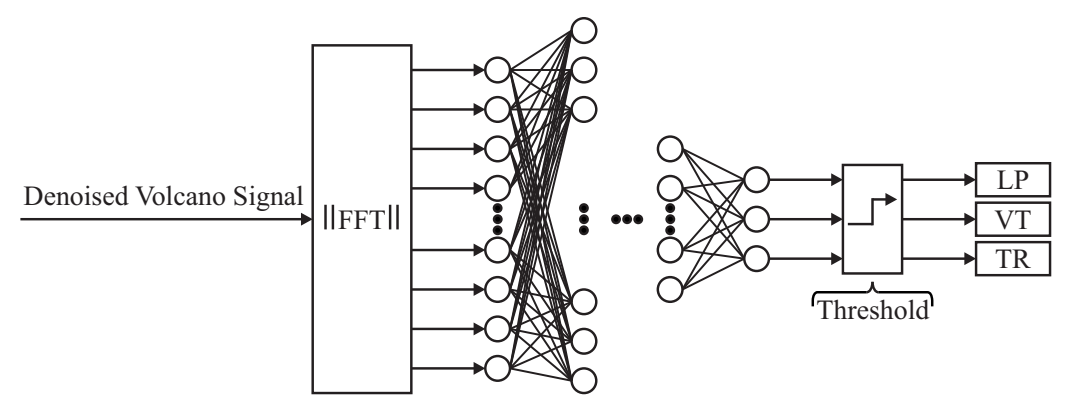

Fig. 6. DNN architecture for event classification 

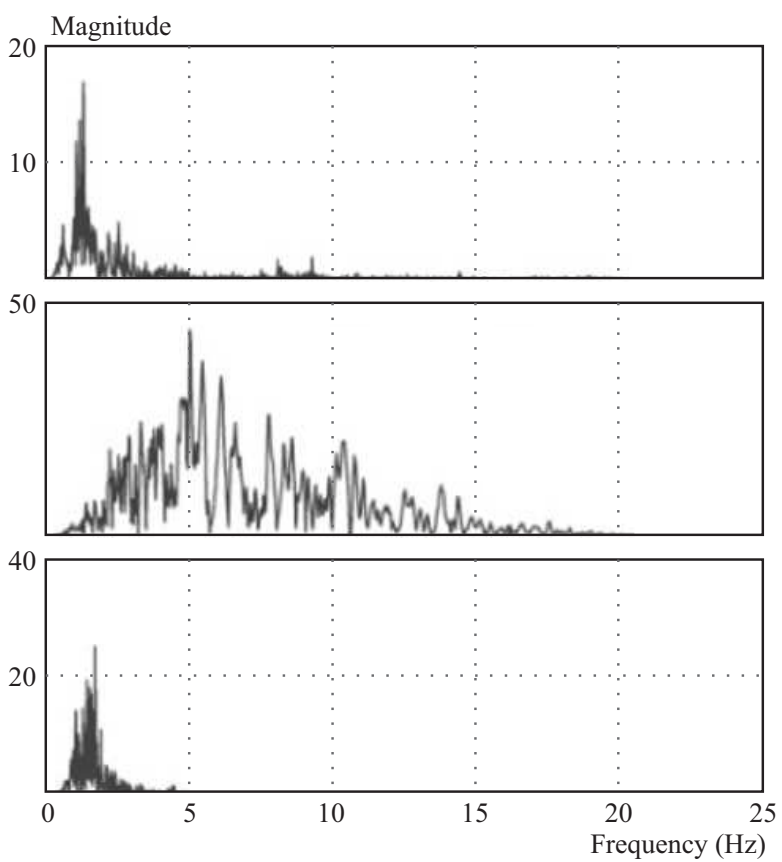

Fig. 7. Frequency spectrum for LP , VT and TR events [41]

where $\theta_{N+1}$ is the parameter set of the output layer. The Back Propagation (BP) algorithm is used to minimize the error between the target data and the DNN output. The above explanation is formally proposed as

$$
\min \vartheta_{D N N}(\theta)=\frac{1}{M} \sum_{m=1}^{M} L\left(y^{m}, d^{m}\right)
$$

where $d^{m}$ is the target data. The parameter set of the network $\theta=\left\{\theta_{1}, \theta_{2}, \ldots, \theta_{N+1}\right\}$ is updates as it follows

$$
\theta_{k+1}=\theta_{k}-\ell \frac{\partial \vartheta_{D N N}}{\partial \theta}
$$

where $\theta_{k+1}$ is the updated weight matrix and $\theta_{k}$ is the current weight matrix of the network. The parameter $\ell$ is the learning rate of the fine tuning in order to obtain a binary output a threshold function was selected to guarantee that only one output will have a positive value, $i e$ $y_{i}=1$ if $y_{i}=\max y$ and $y_{i}=0$ if $y_{i} \neq \max y$.

\subsubsection{Performance indices}

For measuring the performance of the DNN in the classification task different metrics have been used to measure the agreement in the decision between the classification method and the expert. First the Cohen's Kappa coefficient which is a statistic measurement of the inter-rater agreement when independent observers are evaluating the same thing $[39,40]$. The kappa coefficient $(k)$ is defined as

$$
k=\frac{P_{0}-P_{e}}{1-P_{e}}
$$

where $P_{0}$ is the observed agreement between the expert and the classification method and $P_{e}$ is the probability of agreement. The previous variables are mathematically defined as

$$
P_{0}=\sum_{c=1}^{C} p_{c c}, \quad P_{e}=\sum_{c=1}^{C} p_{c} p_{c} .
$$

The results of the classification are shown in a contingency table. The result of the Kappa index can determine if there is a high or low agreement, where the qualitative evaluation of the Kappa index numerical result is given. $k<0$ less than chance agreement, $0.01<k<0.20$ slight agreement, $0.21<k<0.40$ fair agreement, $0.41<k<$ 0.60 moderate agreement, $0.61<k<0.80$ substantial agreement and $0.81<k<0.99$ almost perfect agreement.

Also a binary approach for the performance measurement is carried out. Sensitivity $(S e)$, Specificity $(S p)$, Exactitude $(E x)$ and Error $(E r)$. The first shows if the classifier is good recognizing the positive class, the second shows the ability for recognizing events for a different class, finally, Exactitude and Error show the success and the error of the classifier. The indices explained above are mathematically proposed by

$$
\begin{aligned}
& E x=100 \frac{T P+T N}{n}, \\
& E r=100 \frac{F P+F N}{n}, \\
& S e=100 \frac{T P}{T P+F N}, \\
& S p=100 \frac{T N}{T N+F P}
\end{aligned}
$$

where $T P$ are the true positives, namely, the number of the events that belongs to the positive class and are correctly classified while $T N$ is the number of event of the negative class which are correctly classified. The variables $F P$ and $F N$ are the positive and negative erroneously classified.

\section{Results and discussion}

In this section, we examine denoising, localization and classification methods on recorded signals of Llaima volcano. As we mentioned, MOT, LLA and LAV sensors record mechanical waves produced by the activity of Llaima volcano. These signals have a high level of white and colorful noise. As shown in Fig. 2, the aim of this paper is the localization and classification of recorded signals. Simulations are done using MATLAB software on a PC Core I5, 2.53 GHz CPU and 6 GB RAM.

Events occurring in the depth of earth have different frequency range such. LP is in the range of $[1-5] \mathrm{Hz}, \mathrm{VT}$ is in $[2-15] \mathrm{Hz}$ and $\mathrm{TR}$ is in [1-5]Hz. In addition to this, additive noise in sensors have a broad frequency range. Events have been recorded with frequency sampling $F s=$ $100 \mathrm{~Hz}$ and then highest frequency for noise is $50 \mathrm{~Hz}$. 

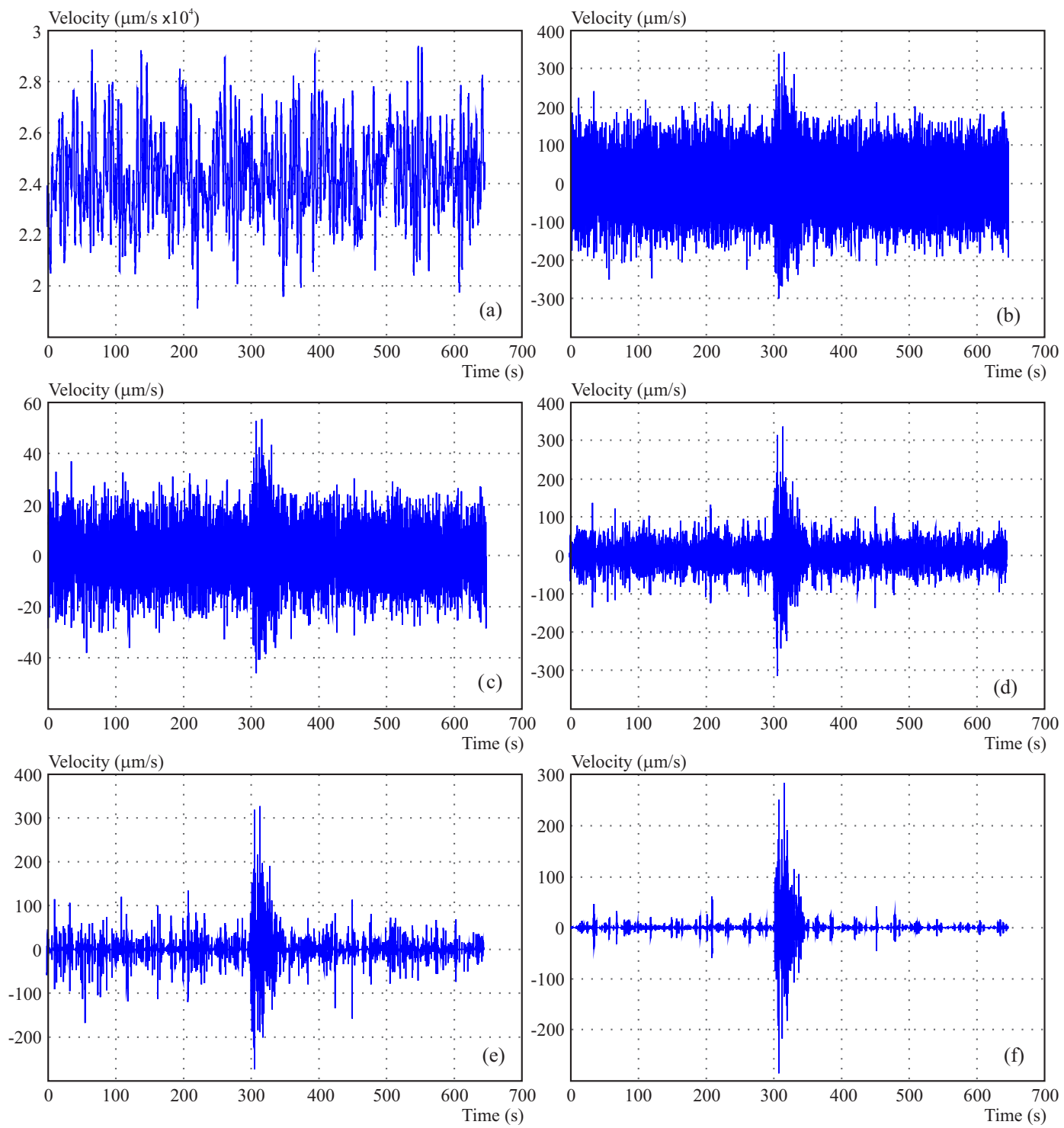

Fig. 8. (a) - Noisy signal and implementation of: (b) - BW, (c) - SS, (d) - WF, (e) - MSS, and (f) - MWF denoising methods on LP event of MOT sensor

Frequency spectrum for LP, VT and TR events has been shown in Fig. 7.

As shown in block diagram Fig. 2, the first step is pre-processing on recorded signals. In first step, we use a Butterworth filter with cut-off frequency $25 \mathrm{~Hz}$. This filter help to remove noise in out of frequency range of events. In following, denoising proposed method in this paper will be examined on recorded signals. SS, WF, MSS and MWF will be evaluated in adverse conditions. Figure 8 , shows obtained results of different denoising methods for LP event in MOT sensor. As shown in Fig. 8, Butterworth method can eliminate noise between 25 to $50 \mathrm{~Hz}$. Also, SS method can remove noise partially but WF can eliminate noise as a considerable value that has better performance rather than SS method. In the following, we implement MSS and MWF denoising methods on recorded signals. We consider $\alpha=1.5$ and $\beta=2.5$ for MSS and $\gamma=1.5$ and $\delta=0.86$ for MWF method. As shown, MWF has better performance than MSS and, it can eliminate a high percentage of noise.

Figure 9, shows the results for denoising methods on VT event. In this case, WF has better results compare to SS method and it could remove noise with high performance. Also, MWF can eliminate an elevated level of noise rather than MSS.

Also, Fig. 10 shows results similar to Figs. 8 and 9. This figure plotted for TR event. In this figure, all denoising methods have been implemented on TR event of sensor MOT. Also in this condition, MWF method have better results compare to other denoising methods. Thus, we use MWF for denoising of recorded signals to prepare enhanced signals for localization and classification.

Table 2, shows the details of output SNR for denoising methods on LP, VT and TR events and for MOT, LAV and LLA sensors. As mentioned, all sensors record signals in $X, Y$ AND $Z$ directions but we just use signal in $x$ direction for simplicity. As shown in Table 2, MWF 

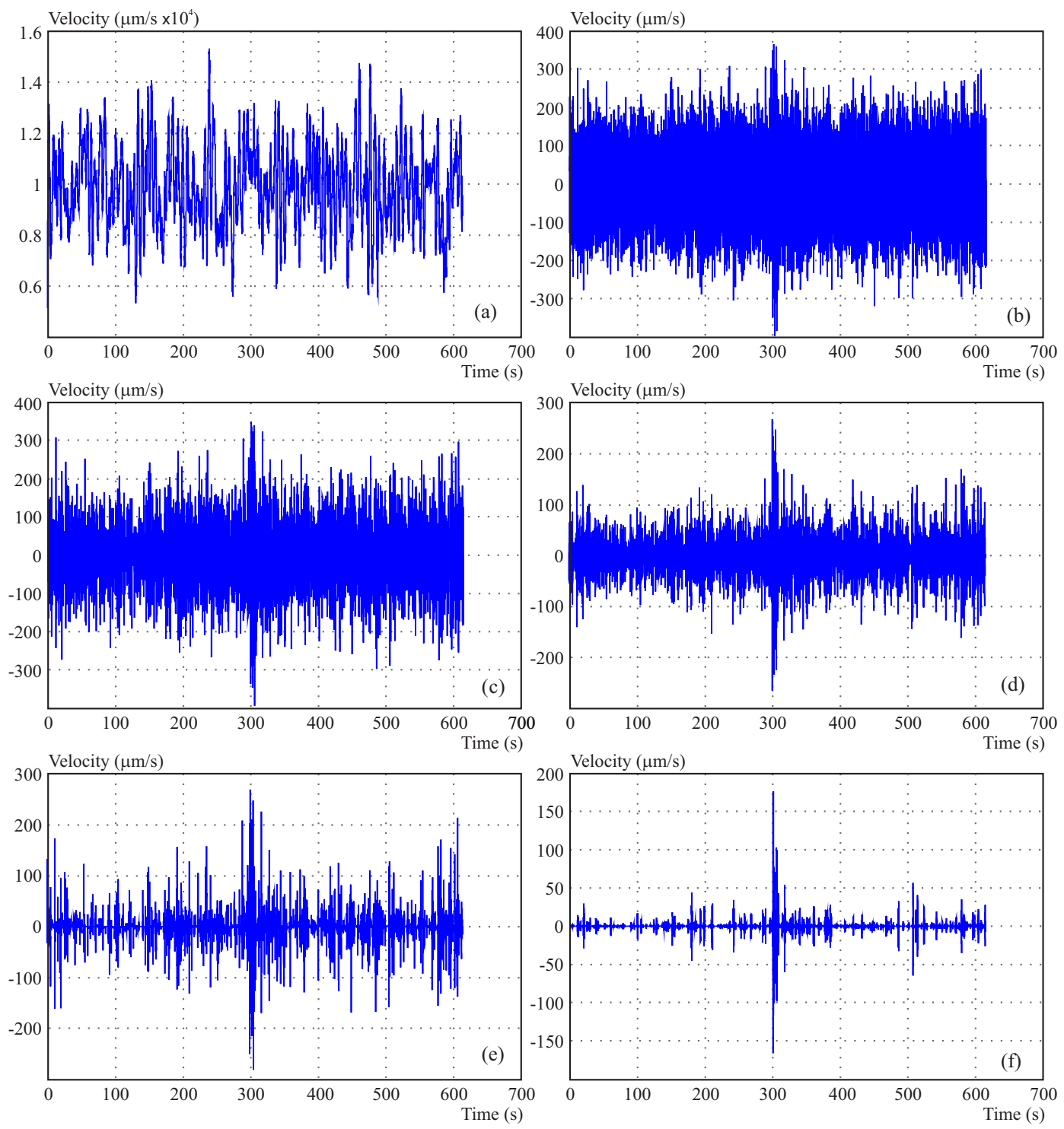

Fig. 9. (a) - Noisy signal and implementation of: (b) - BW, (c) - SS, (d) - WF, (e) - MSS, and (f) - MWF denoising methods on VT event of sensor MOT

denoising method have better results for MOT, LAV and LLA sensors and, for LP, VT and TR events. Based on SNR values, MWF method eliminates a high level of noise with appropriate performance.

Here, we show the results based on GCC-ML function as a localization method for various events. As we mentioned, GCC-ML method localizes the sensor's locations based on TDOA. In this scenario, we use, MOT, LAV and LLA sensors information. Then, one direction is estimated for each pair of sensors.

Figure 11, shows results for a sample LP event. Figure $11(\mathrm{a}-\mathrm{c})$ shows intersection of three estimated directions for sensors in directions $X, Y$ and $Z$ respectively. Finally, figure 11(d) shows estimated points of output $X$, $Y$ and $Z$ direction in the same time to estimate the final point (or we can consider just the final area) for event's location.
In fact, one direction based on GCC-ML function estimated for each pair of sensors and for recorded signal in $Z$ direction for sensors. As shown in Fig. 11(a), a triangular area is formed based on three estimated direction by three sensor pairs that middle point in this area can be considered as event's location for sensors in $X$ directions. Figures 11(b) and 11(c) show similar results for $Y$ and $Z$ directions respectively. Then, one point is estimated for event's location based on GCC-ML function for each sensor in $X, Y$ and $Z$ directions. Figure 11(d) determines the final triangular based on results of Figs. 11(a-c) that this area or the middle point of this area can be considered as an estimated point for LP. Figure 12 shows the results of localization for a sample of VT event. Figure 12(ac) shows the intersection of estimated directions based on GCC-ML function for recorded signals in $X, Y$ and $Z$ directions for VT event respectively. Also, Fig. 12(d) shows the result obtained by sensors in $X, Y$ and $Z$ di- 

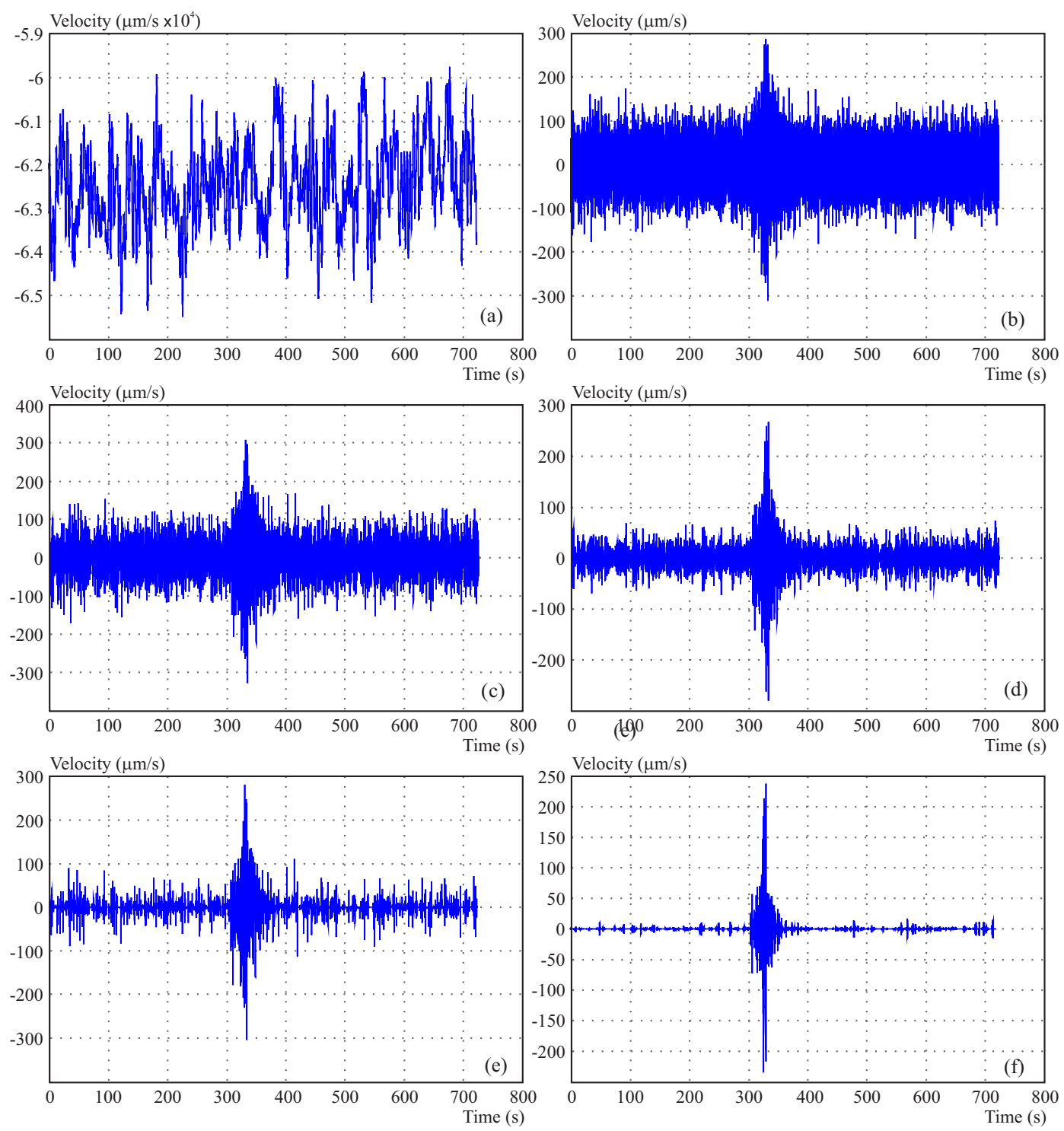

Fig. 10. (a) - Noisy signal and implementation of: (b) - BW, (c) - SS, (d) - WF, (e) - MSS, and (f) - MWF denoising methods on VT event of sensor MOT

Table 2. SNR (dB) results of denoising methods for LP, VT and TR events and for MOT, LAV and L

\begin{tabular}{lccccccccc}
\hline $\begin{array}{l}\text { Input SNR } \\
-3.5 \mathrm{~dB}\end{array}$ & MOT & LAV & LLA & MOT & LAV & LLA & MOT & LAV & LLA \\
\hline BT & -0.52 & -0.12 & 0.25 & -1.53 & -1.07 & -0.86 & -0.24 & 0.08 & 0.36 \\
SS & 2.09 & 2.63 & 2.52 & 1.68 & 1.33 & 2.04 & 2.98 & 3.17 & 3.41 \\
WF & 5.49 & 4.92 & 5.17 & 4.65 & 4.29 & 4.83 & 5.12 & 5.58 & 5.96 \\
MSS & 7.81 & 7.25 & 6.94 & 7.19 & 7.81 & 7.62 & 7.32 & 6.87 & 7.14 \\
MWF & 9.46 & 10.03 & 9.74 & 9.38 & 9.22 & 9.69 & 9.71 & 10.18 & 9.93 \\
\hline
\end{tabular}

rections for VT event that the middle point of triangular area can be considered as event's location.

Figure 13 shows the obtained results of GCC-ML function for a sample of TR event. The intersection for three directions of three sensors pair in $X$ direction is shown in Fig. 13(a). The middle point of this triangular is considered as an estimated point for signals in $X$ direction of sensors. Figures $13(\mathrm{~b}, \mathrm{c})$ show these results for sensors in direction $Y$ and $Z$ respectively. Finally, Fig. 13(d) determines the intersection for estimated points based on sensors in $X, Y$ and $Z$ directions.

The main problem is lack in real value of all events for comparison. Then just we can show the results for proposed method in this paper for localization of VT, LP 

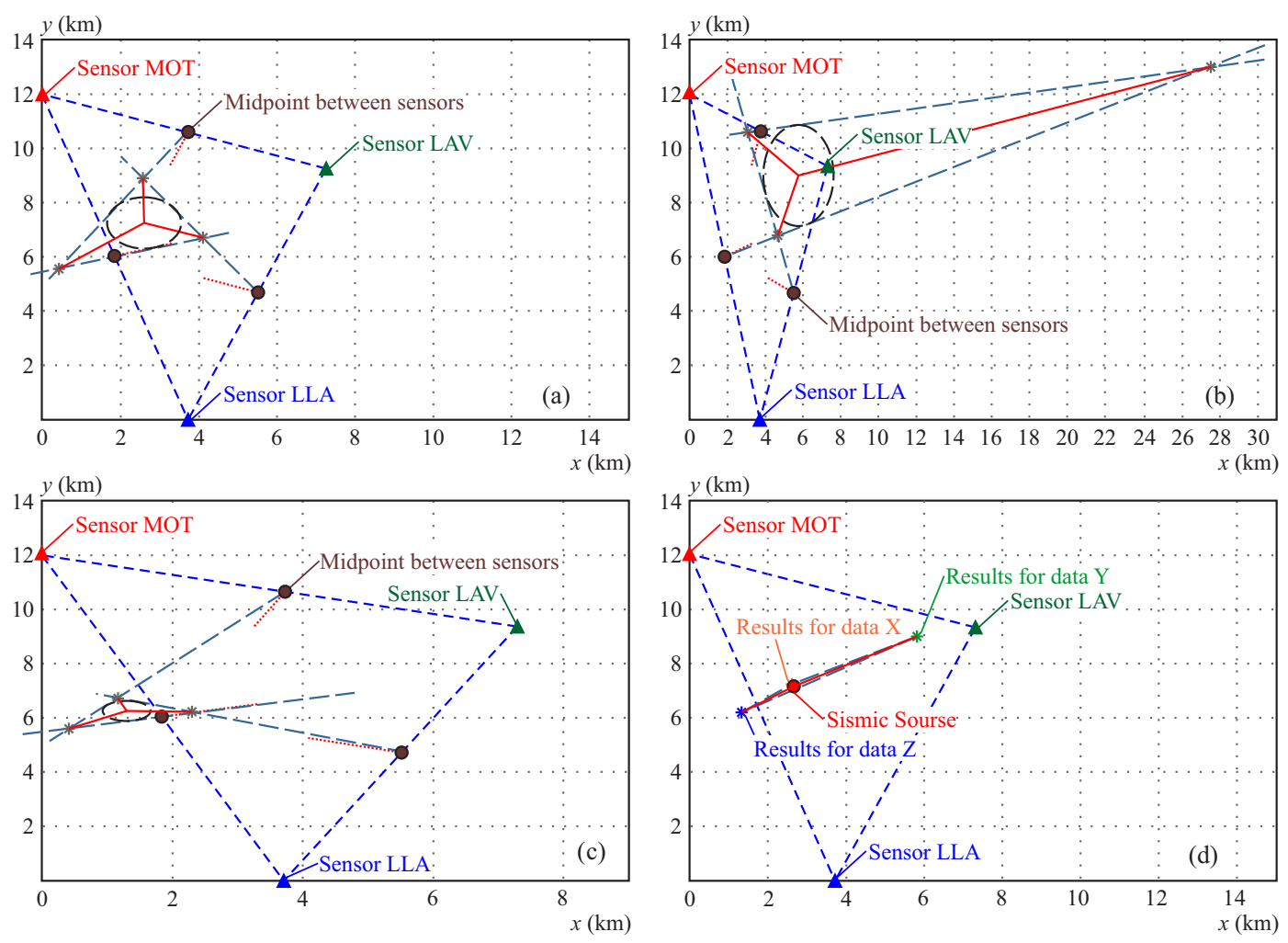

Fig. 11. GCC - based localization results for direction: (a) - X, (b) - Y, (c) - Z, and (d) - intersection between X, Y, Z for estimation the final point for LP event
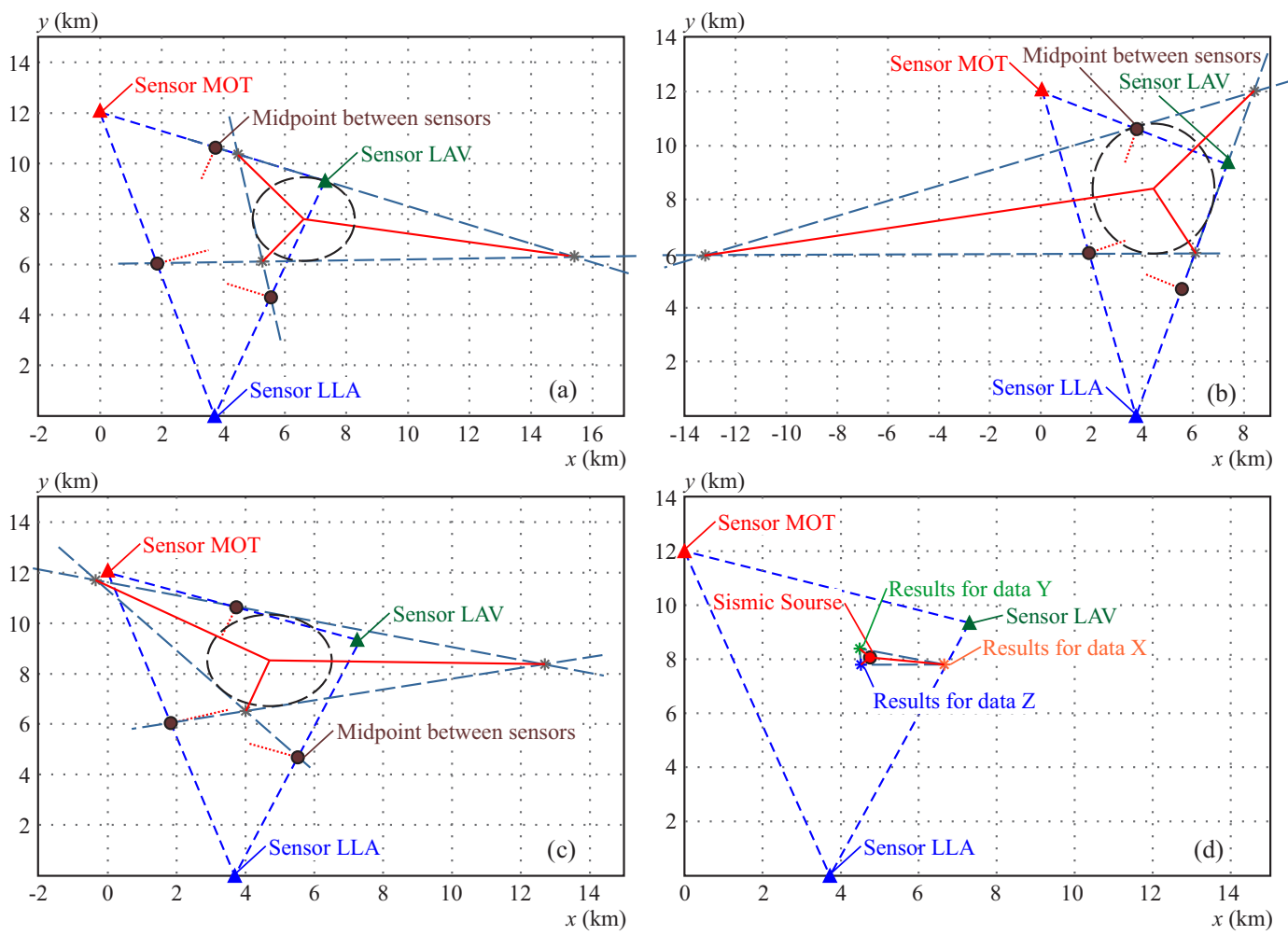

Fig. 12. GCC - based localization results for direction: (a) - X, (b) - Y, (c) - Z, and (d) - intersection between X, Y, Z for estimation the final point for VT event

and VT events. For that reason, we used 20 events for testing the localization algorithm. The results are shown in Fig. 14.
In the classification step, different DNN architectures where tested to find the one which gives higher Kappa index. DNNs with one, two and three hidden layers were 

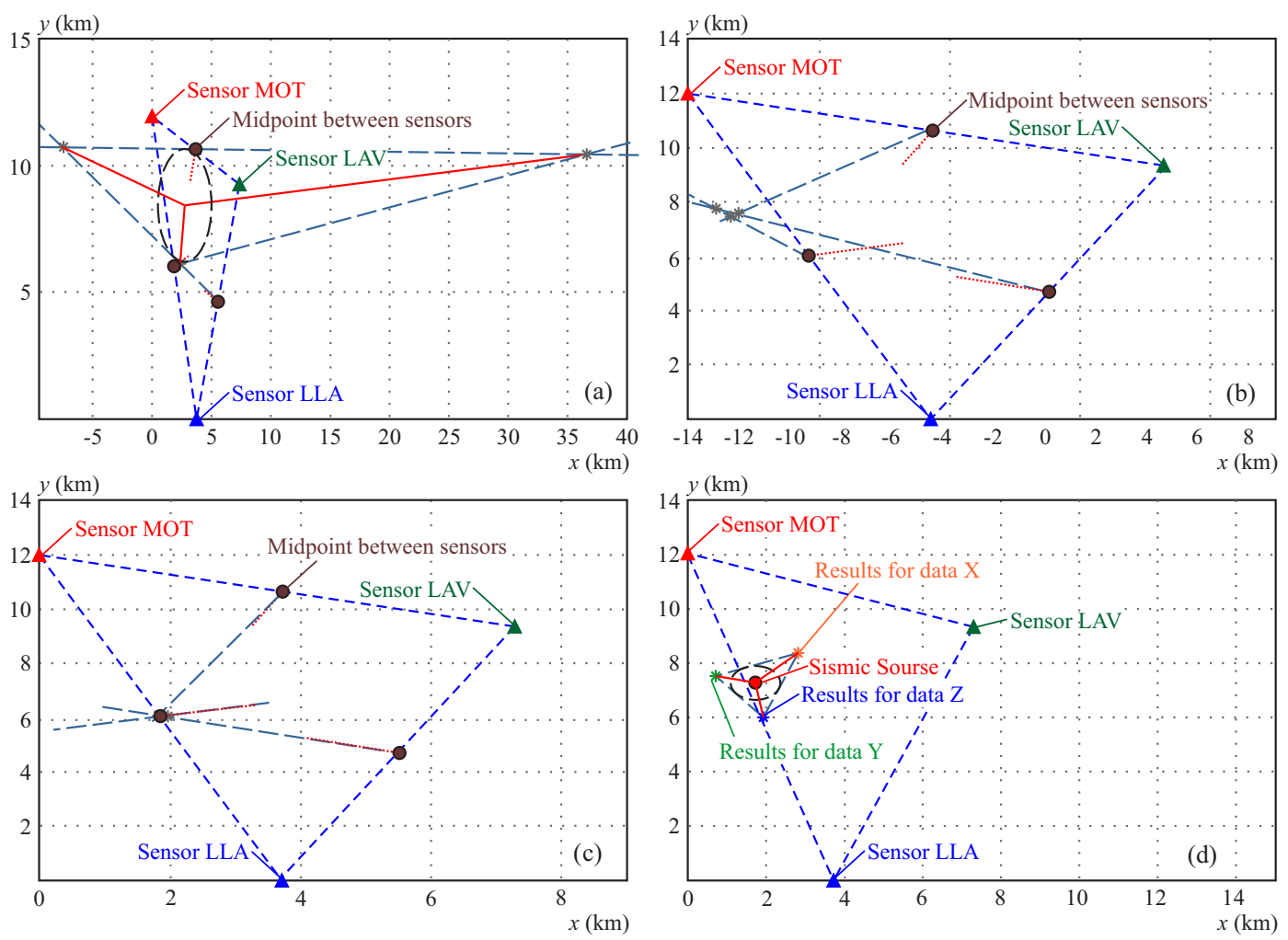

Fig. 13. GCC-based localization results for direction: (a) - X, (b) - Y, (c) - Z, and (d) - intersection between X, Y, Z for estimation the final point for TR event

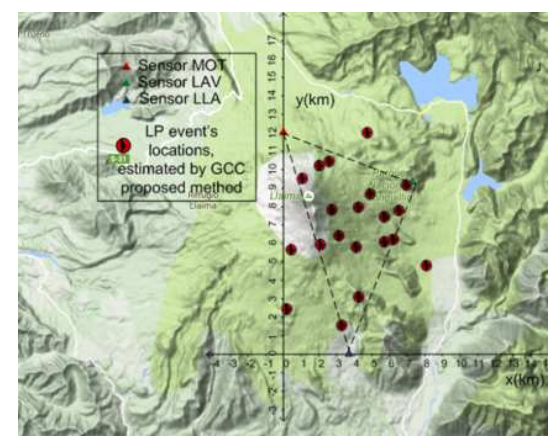

(a)

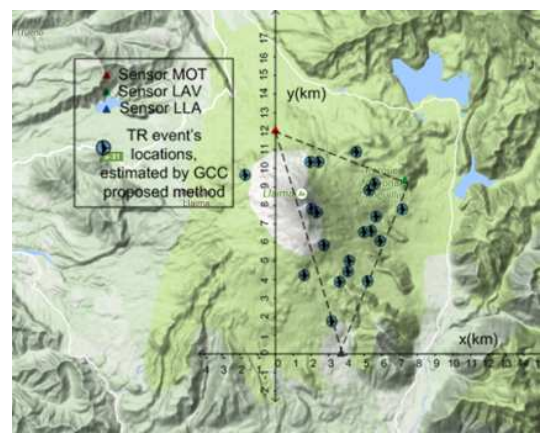

(b)

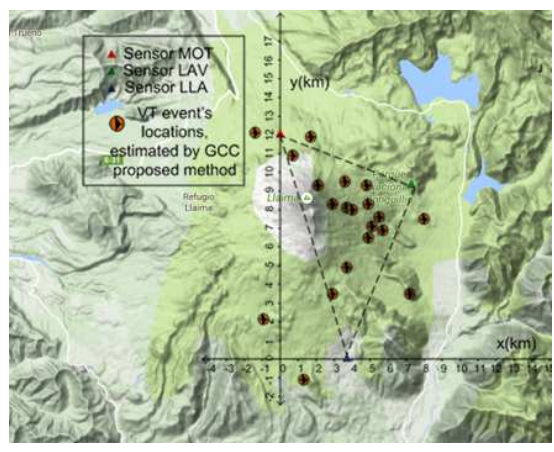

(c)

Fig. 14. Results for proposed method in this paper for localization of: (a) - LP, (b) - TR, and (c) - VT events

tested. The number of neurons on the input layer is 2000 while in the hidden layer the number of neurons was varied from 50 to 200 in step of 50 . The data used corresponds to 268 events, 200 were used for training and 76 for validation, which is considerably less amount of training data compared with the size of the training set used in previous papers $[8,10,42]$. Each event was coded into a binary representation, namely, the DNNs has 3 outputs, each one representing a different event $\left(\mathrm{LP}=\left[\begin{array}{ll}1 & 0\end{array}\right.\right.$ $0], \mathrm{TR}=\left[\begin{array}{lll}0 & 1 & 0\end{array}\right]$ and $\left.\mathrm{VT}=\left[\begin{array}{lll}0 & 0 & 1\end{array}\right)\right]$. The DNN chosen by the above procedure was DNN with 2 hidden layers with 2000 units in the input layer, 100 and 100 neurons on the first and second hidden layers correspondingly and 3 units in the output layer. Deeper architectures are difficult to train when the data set is small. The contingency table of the classifier for validation data is shown in Table 3.For this contingency table the Kappa index is obtained is $k=0.911$.

\section{Conclusions}

The aim of this paper is localization based on recorded events in Llaima volcano in Chile and, classification based on these events. But these signals have a high level of noise because, they have a very low amplitude and they cross different layers of earth. Also, they have seismic records, contained in high frequencies due to ambient noise present (wind, snow, rain, avalanches, etc). The first step in this paper is pre-processing on recorded signals by MOT, LAV and LLA sensors in Llaima volcano. Spectral Subtraction and Wiener Filter were explained as two common denoising methods and then we proposed Modified Spectral Subtraction and Modified Wiener Filter as two modified version of that methods. Those methods were compared on real recorded data for LP, VT and TR events. MWF eliminates an elevated level of noise rather 
than other methods with high performance and it is used to prepare events with low level of noise for localization and classification part. In second step, we use GCC with combination by ML weighted function was selected as a appropriate method for event localization in this paper. This localization method (that is based on TDOA from signals to sensors) is used for localization of various events and, for sensors in $X, Y$ and $Z$ directions. Then, we determine an area based on localization methods for events. In the last part of paper, the aim is classification on recorded signals of LP, VT and TR events. Deep Neural Network is proposed as an appropriate method for classification. This method can classify events with almost perfect agreement with the expert. The proposed method can be applied in a real-time scenario, once the DNN is trained, it performs the classification of the events in matter of seconds.

The Kappa coefficient shows a high agreement between the expert decision and the classifier decision. The binary performance approach is shown in Table 4, Exactitude, Error, Sensitivity and Specificity indices explained in Section 2.4.1.

Table 3. Contingency table for LP, TR and VT events

\begin{tabular}{lcccc}
\hline Clasifier & LP & TR & VT & Total \\
\hline LP & 40 & 0 & 2 & 42 \\
TR & 0 & 14 & 0 & 14 \\
VT & 2 & 0 & 18 & 20 \\
Total & 42 & 14 & 20 & 76 \\
\hline
\end{tabular}

Table 4. Binary performance of the classifier

\begin{tabular}{lccc}
\hline Index & LP & TR & VT \\
\hline $\operatorname{Ex}(\%)$ & 97.36 & 100 & 97.36 \\
$\operatorname{Er}(\%)$ & 5.26 & 0 & 5,26 \\
$\mathrm{Se}(\%)$ & 95.23 & 100 & 90 \\
$\mathrm{Sp}(\%)$ & 94.44 & 100 & 96.55 \\
\hline
\end{tabular}

The binary approach for the performance of the classifier on each event shows that the proposed architecture is able to clustering the event with a highly accurate performance, showing results over $90 \%$ for all the proposed indices.

\section{Acknowledgements}

The authors acknowledge the financial support of the project POSTDOC $_{D} \mathrm{ICYT}$ (No. 041613DA ${ }_{P}$ OSTDOC), Universidad de Santiago de Chile (USACH), Center for multidisciplinary research on signal processing (Project Conicyt/ACT1120), Project USACH/Dicyt No. 061413SG and STIC Amsud Proyect STIC 15STIC-06.

For the work reported in this article, Ali Dehghan Firoozabadi, Fabian Seguel and Ismael Soto motivated and conducted the model design, modification and wrote the paper. David Guevara designed and performed the experiment, analyzed the data. Fernando Huenupan, Millaray Curilem and Luis Franco prepared the data of Observatorio Vulcanolgico de Los Andes del Sur.

\section{Abbreviations}

ANN: Artificial Neural Networks

BP: Back Propagation

DOA: Direction Of Arrival

DNN: Deep Neural Networks

Er: $\quad$ Error

Ex: Exactitude

GCC: Generalized Cross Correlation

LAV: Laguna Verde

LLA: Llaima

LP: $\quad$ Long Period

LPF: Low Pass Filter

ML: $\quad$ Maximum Likelihood

MOT: Motn

MSS: $\quad$ Modified Spectral Subtraction

MWF: Modified Wiener Filter

OVDAS: Observatorio Vulcanolgico De los Andes del Sur

PHAT: PHAse Transform

PSD: $\quad$ Power Spectral Density

Se: $\quad$ Sensitivity

Sp: $\quad$ Specificity

SS: $\quad$ Spectral Subtraction

SVM: $\quad$ Super Vector Machines

TDE: Time Delay Estimation

TDOA: Time Difference Of Arrival

TR: TRemor

VT: $\quad$ Volcano Tectonic

WF: Wiener Filter

\section{REFERENCES}

[1] R. Verdugo, N. Sitar, J. Frost, D et al, "Seismic Performance of Earth Structures during the February 2010 Maule, Chile, Earthquake: Dams, Levees, Tailings Dams, and Retaining Walls", Earthq Spectra 28:S75-S96, doi: 10.1193/1.4000043, 2012.

[2] S. F. L. Watt, T. A. Mather and D. M. Pyle, "Vulcanian Explosion Cycles: Patterns and Predictability", Geology 35:839, doi: 10.1130/G23562A.1, 2007.

[3] V. A. Ramos, "Anatomy and Global Context of the Andes: Main Geologic Features and the Andean Orogenic Cycle", Geol. Soc. Am. Mem. 204:31-65, doi: 10.1130/2009.1204(02), 2009.

[4] L. Lara, G. Orozco, A. Amigo and C. Silva, "Peligros Volcánicos de Chile", Carta Geológica de Chile 1, (2011).

[5] "Observatorio Volcanológico, de, Los-Andes del Sur (OVDAS)" Sernageomin.

[6] L. E. Franco, J. L. Palma, F. Gil-cruz and J. J. San-Martín, "La Activeidad Sísmica Asociada a Erupciones Estrombolianas Violentas en el volcán Llaima, Chile (2007-2010)", III Congr. Latinoam. Sismol., 2014

[7] M. Muñoz, "Eruption Patterns of the Chilean Volcanoes Villarrica, Llaima, and Tupungatito", Pure Appl. Geophys. PAGEOPH 121:835-852, doi: 10.1007/BF02590184, 1983.

[8] M. Curilem, J. Vergara, C. S. Martin et al, "Pattern Recognition Applied to Seismic Signals of the Llaima Volcano (Chile): An Analysis of the Events' Features", J. Volcanol Geotherm Res. 282:134-147, doi: 10.1016/j.jvolgeores.2014.06.004, 2014.

[9] B. A. Chouet and R. S. Matoza, "A Multi-Decadal View of Seismic Methods for Detecting Precursors of Magma Movement and Eruption", J. Volcanol Geotherm Res. 252:108-175, doi: 10.1016/j.jvolgeores.2012.11.013, 2013.

[10] G. Curilem, J. Vergara, G. Fuentealba et al, "Classification of Seismic Signals at Villarrica Volcano (Chile) using Neural 
Networks and Genetic Algorithms", J. Volcanol Geotherm. Res 180:1-8, doi: 10.1016/j.jvolgeores.2008.12.002, 2009.

[11] S. Falsaperla, S. Graziani, G. Nunnari and S. Spampinato, "Automatic Classification of Volcanic Earthquakes by using Multi-Layered Neural Networks", Nat. Hazards, doi: 10.1007/BF00215816, 1996.

[12] B. Chouet, Volcanic Seismology, doi: 10.1007/978-3-642-77008-1, 1992.

[13] P. Podder, M. M. Hasan, M. R. Islam and M. Sayeed, "Design and Implementation of Butterworth, Chebyshev-I and Elliptic Filter for Speech Signal Analysis", nt. J. Comput. Appl. 98:12-18.

[14] Y. Lu and P. C. Loizou, "A Geometric Approach to Spectral Subtraction", Speech Commun., 50:453-466, doi: 10.1016/j.specom.2008.01.003, 2008.

[15] Z. Ghahramani, "An Introduction to Hidden Markov Models and Bayesian Networks", Int. J. Pattern Recognit. Artif. Intell. 15:9-42, doi: 10.1142/S0218001401000836, 2001.

[16] A. L. Swindlehurst and T. Kailath, "Aperformance Analysis of Subspace-Based Methods the Presence of Model Errors. I. The MUSIC Algorithm", IEEE Trans. Signal Process. 40:1758-1774, doi: 10.1109/78.143447.

[17] W. H. K. Lee, J. C. Lahr and C. M. Valdes, International Handbook of Earthquake and Engineering Seismology, Int. Geophys. Elsevier, pp. 1641-1642, 2003

[18] J. C. Lahr and J. A. Snoke, International Handbook of Earthquake and Engineering Seismology, Int. Geophys. Elsevier, pp. 1617-1618, 2003.

[19] F. W. Klein, International Handbook of Earthquake and Engineering Seismology, Int. Geophys. Elsevier, pp. 1619-1620, 2003

[20] N. Madhu and R. Martin, "Source Localization with Microphone Arrays", Adv. Digit Speech Transm., 135-170, doi: 10.1002/9780470727188, 2008.

[21] H. Krim and M. Viberg, "Two Decades of Array Signal Processing Research: The Parametric Approach", IEEE Signal Process Mag., 13:67-94, doi: 10.1109/79.526899, 1996.

[22] P. Stoica, Introduction to Spectral Analysis, Prentice Hall, 1997.

[23] R. Schmidt, "Multiple Emitter Location and Signal Parameter Estimation", IEEE Trans. Antennas Propag., 34:276-280, doi 10.1109/TAP.1986.1143830, 1986.

[24] R. Roy and T. Kailath, "ESPRIT-Estimation of Signal Parameters via Rotational Invariance Techniques", IEEE Trans. Acoust 37:984-995, doi: 10.1109/29.32276, 1989.

[25] M. Morf, "The Signal Subspace Approach for Multiple WideBand Emitter Location", IEEE Trans. Acoust. 31:1502-1522, doi: 10.1109/TASSP.1983.1164233, 1983.

[26] B. Kwon, Y. Park and Y. S. Park, "Multiple Sound Sources Localization using the Spatially Mapped GCC Functions", 773-1776.

[27] S. Kuifan, O. Yuehui and D. Jiwen, "Tsinghua Science and Technology", Tsinghua Sci. Technol. 2:853-855, 1997.

[28] S. Kiranyaz, T. Ince and M. Gabbouj, "Real-Time Patient-Specific ECG Classification by 1-D Convolutional Neural Networks", IEEE Trans. Biomed. Eng. 63:664-75, doi: 10.1109/TBME.2015.2468589, 2016.
[29] I. Mcloughlin, H. Zhang, Z. Xie et al, "Robust Sound Event Classification Using Deep Neural Networks", IEEE/ACM Trans Audio, Speech, Lang. Process 23:540-552, doi: 10.1109/TASLP.2015.2389618, 2015.

[30] I. Monedero, C. Leon, J. Ropero et al, "Classification of Electrical Disturbances Real Time Using Neural Networks", IEEE Trans. Power Deliv. 22:1288-1296, doi: 10.1109/TPWRD.2007.899522, 2007

31] Shengnan-Hao, Jing-Hu, Songyin-Liu et al, "Improved SVM Method for Internet Traffic Classification based on Feature Weight Learning", 2015 Int. Conf. Control. Autom. Inf. Sci. IEEE, pp. 102-106, 2015.

[32] Y. Bengio, P. Lambling, D. Popovici and H. Larochelle, "Greedy Layer-Wise Training of Deep Networks", Adv. Neural. Inf. Process. Syst. 19, MIT Press, Vancouver, B.C., Canada, pp. 153-160, 2007.

[33] G. E. Hinton and R. R. Salakhutdinov, "Reducing the Dimensionality of Data with Neural Networks", Science 313:504-7, doi: 10.1126/science.1127647, 2006

34] J. Niu, Y. Qian and K. Yu, "Acoustic Emotion Recognition using Deep Neural Network", 9th Int. Symp. Chinese Spok. Lang. Process. IEEE, pp. 128-132, 2014.

35] A. Graves, A. Mohamed and G. Hinton, "Speech Recognition with Deep Recurrent Neural Networks", 2013 IEEE Int. Conf. Acoust. Speech Signal Process. IEEE, pp. 6645-6649, 2013.

36] C. Bentes, D. Velotto and S. Lehner, "Target Classification Oceanographic SAR Images with Deep Neural Networks: Architecture and Initial Results", 2015 IEEE Int. Geosci. Remote Sens. Symp. IEEE, pp. 3703-3706, 2015.

37] L. Chmelka and J. Kozumplik, "Wavelet-Based Wiener Filter for Electrocardiogram Signal Denoising", Comput. Cardiol. 2005. IEEE, pp. 771-774, 2005.

[38] D. Firoozabadi and A. Abutalebi, "A Novel Nested Circular Microphone Array and Subband Processing-Based System for Counting and DOA Estimation of Multiple Simultaneous Speakers", Circuits, Syst. Signal Process. 35:573-601, doi: 10.1007/s00034-015-0077-6, 2015.

[39] A. J. Viera and J. M. Garrett, "Understanding Interobserver Agreement: The Kappa Statistic", Fam. Med. 37:360-3, 2005.

[40] J. R. Landis and, G. G. Koch, "The Measurement of Observer Agreement for Categorical Data", Biometrics 33:159, doi: 10.2307/2529310, 1977.

[41] M. Moreno, D. Melnick, M. Rosenau et al, "Toward Understanding Tectonic Control on the Mw 8.82010 Maule Chile Earthquake", Earth Planet Sci. Lett. 321-322:152-165, doi: 10.1016/j.epsl.2012.01.006, 2012

[42] M. Curilem, F. Huenupan, D. Beltrán et al, "Pattern Recognition Applied to Seismic Signals of Llaima Volcano (Chile): An Evaluation of Station-Dependent Classifiers", J. Volcanol. Geotherm Res., 315:15-27, doi: 10.1016/j.jvolgeores.2016.02.006, 2016 .

Received 13 April 2017 